\title{
Anyon exciton revisited: Exact solutions for a few-particle system
}

\author{
D.G.W. Parfitt and M.E. Portnoi* \\ School of Physics, University of Exeter, Stocker Road, Exeter EX4 4QL, United Kingdom
}

(Received 20 February 2003; published 3 July 2003)

\begin{abstract}
The anyon exciton model is generalized to the case of a neutral exciton consisting of a valence hole and an arbitrary number $N$ of fractionally charged quasielectrons (anyons). A complete set of exciton basis functions is obtained and these functions are classified using a result from the theory of partitions. Expressions are derived for the interparticle interaction matrix elements of a six-particle system $(N=5)$, which describes an exciton against the background of an incompressible quantum liquid with filling factor $\nu=2 / 5$. Several exact results are obtained in a boson approximation, including the binding energy of a $(N+1)$-particle exciton with zero in-plane momentum and zero internal angular momentum.
\end{abstract}

DOI: 10.1103/PhysRevB.68.035306

PACS number(s): 73.43.Cd, 71.35.Ji, 71.10.Pm

\section{INTRODUCTION}

The anyon exciton model (AEM), which considers a neutral exciton made up of a valence hole and several fractionally charged quasielectrons with fractional statistics (anyons), was proposed a decade ago by Rashba and Portnoi. $^{1}$ It was later developed ${ }^{2}$ to model incompressible quantum liquids (IQL's) with filling factors $\nu=1 / 3$ and $2 / 3$, and is valid at large separation between the hole and the two-dimensional electron gas (2DEG), so that the Coulomb field of the hole does not destroy the IQL. The AEM was motivated by experiments on intrinsic photoluminescence (PL) in the fractional quantum Hall regime ${ }^{3-5}$ that showed a pronounced double-peak structure in emission spectra, and it has provided major insights into the role of electron-hole separation in determining the optical spectra. The model yields multiple-branch energy spectra and gives a full classification of states for a four-particle system. It also predicts an increase in ground-state angular momentum and flattening of the corresponding dispersion curve with increasing layer separation. The AEM has also shown good agreement for intermediate electron-hole separation with numerical finitesize calculations by Apalkov and co-workers. 6,7

At the time when the model was proposed, most experiments on intrinsic PL were carried out at constant carrier density, with the filling factor changed by varying the magnetic field. However, the AEM is valid for fixed fractional filling factors only. More recent experimental techniques enable the 2DEG density in the PL experiments to be changed using applied gate voltages ${ }^{8-11}$ or by varying the light intensity. ${ }^{12}$ Thus, it is now possible to manipulate the effective electron-hole separation (in units of magnetic length) while keeping the filling factor constant. So far, charged excitons have been observed for small electron-hole separation, but for large separations it is predicted that neutral anyon excitons should be observed.

These recent developments have rekindled our interest in the theoretical treatment of spatially separated electron-hole systems. In this paper we address some important mathematical features of the AEM. In Sec. II we derive a general few-particle wave function for a neutral exciton consisting of a valence hole and $N$ anyons. In Sec. III we move to consider interaction matrix elements for a six-particle anyon exciton, which should be useful for analysis of intrinsic PL experiments at $\nu=2 / 5$. (Note that there has been some debate as to whether the ground state at $\nu=1 / 5$ is actually an IQL; see, e.g., Ref. 13 and references therein.) In Sec. IV, exact results for the general $(N+1)$-particle case are obtained and discussed. It is shown that the exciton remains bound even at large separations between the 2DEG and the hole.

\section{EXCITON BASIS FUNCTIONS}

\section{A. Preliminaries}

We consider an exciton consisting of a valence hole with charge $+e$ and $N$ anyons with charge $-e / N$ and statistical factor $\alpha$. The hole and anyons reside in two different layers, separated by a distance of $h$ magnetic lengths, and are subject to a magnetic field $\mathbf{H}=H \hat{\mathbf{z}}$ perpendicular to their planes of confinement. Unless otherwise stated, we shall assume that the hole and the quasielectrons are in their corresponding lowest Landau levels.

To simplify the description of the exciton, the hole and anyons may be considered as moving in the same plane with coordinates $\mathbf{r}_{h}$ and $\mathbf{r}_{j}$, respectively, and the layer separation $h$ can be introduced later when considering the anyon-hole interaction.

An exciton consisting of a hole and $N$ anyons, all in the lowest Landau level, will have a total of $N+1$ degrees of freedom. As the exciton is neutral, we can assign it an inplane momentum $\mathbf{k}$, which absorbs two of these degrees of freedom. For $N \geqslant 2$ the exciton will have $N-1$ internal degrees of freedom, which results in internal quantum numbers and a multiple-branch energy spectrum.

\section{B. Derivation of basis functions}

At this stage we consider the particles as noninteracting and introduce interactions later as necessary. We can therefore write the $(N+1)$-particle Hamiltonian as

$$
\hat{H}_{0}=\frac{1}{2 m_{h}}\left(\hat{\mathbf{p}}_{h}-\frac{q_{h} \mathbf{A}}{c}\right)^{2}+\sum_{j=1}^{N} \frac{1}{2 m_{a}}\left(\hat{\mathbf{p}}_{j}-\frac{q_{a} \mathbf{A}}{c}\right)^{2}
$$

where $q_{h}=+e$ and $q_{a}=-e / N$ are the hole and anyon charges, respectively. 


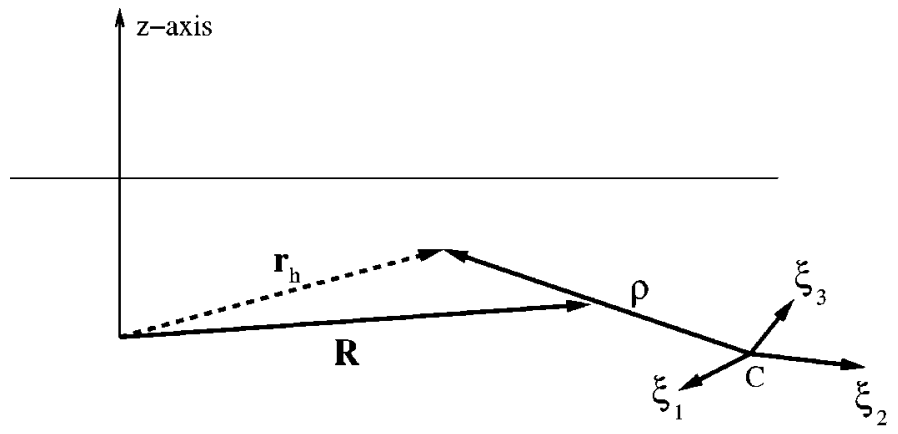

Choosing the symmetric gauge

$$
\mathbf{A}=\frac{1}{2}[\mathbf{H} \times \mathbf{r}]
$$

and scaling all distances with the magnetic length $l_{H}$ $=(c \hbar / e H)^{1 / 2}$, we obtain

$$
\begin{aligned}
\hat{H}_{0}= & \frac{1}{2 m_{h}}\left(\frac{1}{i} \boldsymbol{\nabla}_{h}-\left[\hat{\mathbf{z}} \times \mathbf{r}_{h}\right]\right)^{2} \\
& +\sum_{j=1}^{N} \frac{1}{2 m_{a}}\left(\frac{1}{i} \nabla_{j}+\frac{1}{N}\left[\hat{\mathbf{z}} \times \mathbf{r}_{j}\right]\right)^{2},
\end{aligned}
$$

where, as usual, $e, \hbar, c$, and the dielectric constant are assumed equal to unity.

We now introduce the following new coordinates (see Fig. 1):

$$
\begin{gathered}
\mathbf{R}=\frac{1}{2}\left(\mathbf{r}_{h}+\frac{1}{N} \sum_{j=1}^{N} \mathbf{r}_{j}\right), \quad \boldsymbol{\rho}=\mathbf{r}_{h}-\frac{1}{N} \sum_{j=1}^{N} \mathbf{r}_{j}, \\
\boldsymbol{\xi}_{j}=\mathbf{r}_{j}-\frac{1}{N} \sum_{l=1}^{N} \mathbf{r}_{l},
\end{gathered}
$$

together with the complex coordinates $\zeta_{j}=\xi_{x j}+i \xi_{y j}$. Note also the following constraint on these coordinates:

$$
\sum_{j=1}^{N} \boldsymbol{\xi}_{j}=\sum_{j=1}^{N} \zeta_{j}=0
$$

The fact that the variables $\boldsymbol{\xi}_{j}$ are not independent means that derivatives with respect to these variables are not defined. However, it is possible to introduce the derivatives $\boldsymbol{\nabla}_{\xi_{j}}$, which treat the variables $\boldsymbol{\xi}_{j}$ as if they were independent, ${ }^{14}$ by working in a space of higher dimensionality and then considering a submanifold in this space defined by constraint (5).

Using the chain rule, we may now rewrite the derivatives of a function $f$ with respect to $\mathbf{r}_{j}$ as
FIG. 1. Two-dimensional coordinate system for hole and several quasielectrons (anyons). The hole is considered as being in the same plane as the anyons, with layer separation introduced when considering the anyon-hole interaction. $C$ indicates the center of negative charge.

The old coordinates can be expressed in terms of the new ones as follows:

$$
\mathbf{r}_{h}=\mathbf{R}+\frac{\boldsymbol{\rho}}{2}, \quad \mathbf{r}_{j}=\mathbf{R}-\frac{\boldsymbol{\rho}}{2}+\boldsymbol{\xi}_{j}
$$

and the corresponding derivatives as

$$
\begin{gathered}
\boldsymbol{\nabla}_{h}=\frac{1}{2} \boldsymbol{\nabla}_{\mathbf{R}}+\boldsymbol{\nabla}_{\boldsymbol{\rho}}, \\
\boldsymbol{\nabla}_{j}=\frac{1}{2 N} \boldsymbol{\nabla}_{\mathbf{R}}-\frac{1}{N} \boldsymbol{\nabla}_{\boldsymbol{\rho}}+\boldsymbol{\nabla}_{\xi_{j}}-\frac{1}{N} \sum_{l=1}^{N} \boldsymbol{\nabla}_{\xi_{l}} .
\end{gathered}
$$

We now write the Hamiltonian (3) in terms of the new variables. Using Eqs. (6)-(9) we obtain

$$
\hat{H}_{0}=\hat{H}_{e x c}+\hat{H}_{\xi},
$$

where

$$
\begin{aligned}
\hat{H}_{\text {exc }}= & \frac{1}{2 m_{h}}\left\{\frac{1}{2 i} \boldsymbol{\nabla}_{\mathbf{R}}+\frac{1}{i} \boldsymbol{\nabla}_{\boldsymbol{\rho}}-\frac{1}{2}\left[\hat{\mathbf{z}} \times\left(\mathbf{R}+\frac{\boldsymbol{\rho}}{2}\right)\right]\right\}^{2} \\
& +\frac{1}{2 N m_{a}}\left\{\frac{1}{2 i} \boldsymbol{\nabla}_{\mathbf{R}}-\frac{1}{i} \boldsymbol{\nabla}_{\boldsymbol{\rho}}+\frac{1}{2}\left[\hat{\mathbf{z}} \times\left(\mathbf{R}-\frac{\boldsymbol{\rho}}{2}\right)\right]\right\}^{2},
\end{aligned}
$$

and

$$
\hat{H}_{\xi}=\frac{1}{2 m_{a}} \sum_{j=1}^{N}\left\{\frac{1}{i} \boldsymbol{\nabla}_{\xi_{j}}-\frac{1}{N i} \sum_{l=1}^{N} \boldsymbol{\nabla}_{\xi_{l}}+\frac{1}{2 N}\left[\hat{\mathbf{z}} \times \boldsymbol{\xi}_{j}\right]\right\}^{2} .
$$

Note that Eq. (12) was obtained by applying constraint (5).

The first part $\hat{H}_{\text {exc }}$ is similar to that for a standard diamagnetic exciton, ${ }^{15,16}$ with the electron mass replaced with $N$ anyon masses. The eigenfunctions of this operator can be written straightforwardly as 


$$
\Psi_{e x c}(\mathbf{R}, \boldsymbol{\rho})=\exp \{i \mathbf{k} \cdot \mathbf{R}+i \hat{\mathbf{z}} \cdot[\mathbf{R} \times \boldsymbol{\rho}] / 2\} \Phi(\boldsymbol{\rho}),
$$

where $\Phi(\boldsymbol{\rho})$ satisfies the equation

$$
\begin{aligned}
& \left(\frac{1}{2 m_{h}}\left\{\frac{1}{i} \boldsymbol{\nabla}_{\boldsymbol{\rho}}-\frac{[\hat{\mathbf{z}} \times(\boldsymbol{\rho}-\mathbf{d})]}{2}\right\}^{2}\right. \\
& \left.\quad+\frac{1}{2 N m_{a}}\left\{\frac{1}{i} \boldsymbol{\nabla}_{\boldsymbol{\rho}}+\frac{[\hat{\mathbf{z}} \times(\boldsymbol{\rho}-\mathbf{d})]}{2}\right\}^{2}\right) \Phi=E_{\text {exc }} \Phi
\end{aligned}
$$

Here, $\mathbf{d}=\mathbf{k} \times \hat{\mathbf{z}}$ is the exciton dipole moment. Note that Eq. (13) does not contain the mass-dependent phase factor that appears in the wave function in Ref. 16. This is due to our choice of the center of charge of the exciton for our coordinate $\mathbf{R}$, rather than the center of mass chosen in Refs. 15 and 16. Thus, the quantum number $\mathbf{k}$ entering Eq. (13) represents the momentum of the geometrical center of the exciton.

The eigenfunctions are then

$$
\Phi_{n m}=|\boldsymbol{\rho}-\mathbf{d}|^{|m|} L_{n}^{|m|}\left\{(\boldsymbol{\rho}-\mathbf{d})^{2} / 2\right\} \exp \left\{i m \phi-(\boldsymbol{\rho}-\mathbf{d})^{2} / 4\right\},
$$

where $\phi$ is the azimuthal angle of the vector $(\boldsymbol{\rho}-\mathbf{d})$ and $L_{n}^{m}(z)$ are the associated Laguerre polynomials. The corresponding eigenvalues are

$$
E_{\text {exc }}=\frac{1}{m_{h}}\left(n+\frac{|m|-m+1}{2}\right)+\frac{1}{N m_{a}}\left(n+\frac{|m|+m+1}{2}\right) .
$$

Note that in Eqs. (15) and (16) we no longer restricted ourselves to the lowest Landau level for the hole and anyons. For the ground-state $(n, m=0)$, Eq. (13) reduces to

$$
\Psi_{e x c}(\mathbf{R}, \boldsymbol{\rho})=\exp \left\{i \mathbf{k} \cdot \mathbf{R}+i \hat{\mathbf{z}} \cdot[\mathbf{R} \times \boldsymbol{\rho}] / 2-(\boldsymbol{\rho}-\mathbf{d})^{2} / 4\right\}
$$

which depends on the quantum number $\mathbf{k}$ alone.

We now move to consider the term $\hat{H}_{\xi}$ in Eq. (12), which may be divided into two parts:

$$
\hat{H}_{1 \xi}=\frac{1}{2 m_{a}}\left(1-\frac{1}{N}\right) \sum_{j=1}^{N}\left(\frac{1}{i} \nabla_{\xi_{j}}+\frac{1}{2 N}\left[\hat{\mathbf{z}} \times \boldsymbol{\xi}_{j}\right]\right)^{2},
$$

and

$$
\begin{aligned}
\hat{H}_{2 \xi}= & \frac{1}{2 N m_{a}} \sum_{j=1}^{N}\left\{-\nabla_{\boldsymbol{\xi}_{j}}^{2}+\frac{1}{N i}\left[\hat{\mathbf{z}} \times \boldsymbol{\xi}_{j}\right] \cdot\left(\boldsymbol{\nabla}_{\boldsymbol{\xi}_{j}}-\sum_{l=1}^{N} \boldsymbol{\nabla}_{\xi_{l}}\right)\right. \\
& \left.+\frac{1}{N^{2}}\left|\boldsymbol{\xi}_{j}\right|^{2}+2 \boldsymbol{\nabla}_{\xi_{j}} \cdot\left(\sum_{l=1}^{N} \boldsymbol{\nabla}_{\boldsymbol{\xi}_{l}}\right)-\frac{1}{N}\left(\sum_{l=1}^{N} \boldsymbol{\nabla}_{\boldsymbol{\xi}_{l}}\right)^{2}\right\} .
\end{aligned}
$$

The eigenfunctions of the ground state of $\hat{H}_{1 \xi}$ can then be written in terms of complex coordinates as

$$
\Phi_{\xi}=\prod_{j}\left(\bar{\zeta}_{j}\right)^{\beta_{j}} \exp \left\{-\left|\zeta_{j}\right|^{2} / 4 N\right\}
$$

with the corresponding eigenvalues

$$
E_{\xi}=\frac{1}{2 m_{a}}\left(1-\frac{1}{N}\right) \text {. }
$$

If we now apply constraint (5), we find that the function $\Phi_{\xi}$ is also an eigenfunction of $\hat{H}_{2 \xi}$, with a corresponding eigenvalue of zero, i.e.,

$$
\hat{H}_{2 \xi} \Phi_{\xi} \propto\left(\sum_{j=1}^{N} \xi_{j}\right)^{2} \Phi_{\xi}=0
$$

A calculation of the total energy of the ground state gives

$$
E=E_{e x c}+E_{\xi}=\frac{1}{2 m_{h}}+\frac{1}{2 m_{a}}=\frac{1}{2}\left(\omega_{h}+N \omega_{a}\right),
$$

which is indeed the ground-state energy of the original $(N$ +1 )-particle Hamiltonian in Eq. (3).

The most general form for the ground-state eigenfunction of $\hat{H}_{0}$ is

$$
\begin{aligned}
\Psi\left(\mathbf{R}, \boldsymbol{\rho},\left\{\zeta_{i}\right\}\right)= & \exp \left\{i \mathbf{k} \cdot \mathbf{R}+i \hat{\mathbf{z}} \cdot[\mathbf{R} \times \boldsymbol{\rho}] / 2-(\boldsymbol{\rho}-\mathbf{d})^{2} / 4\right\} \\
& \times F\left(\ldots, \bar{\zeta}_{i}, \ldots\right) \prod_{p} \exp \left\{-\left|\zeta_{p}\right|^{2} / 4 N\right\},
\end{aligned}
$$

where $F$ must only be a function of the complex conjugates $\bar{\zeta}_{i}$. We choose $F$ as follows to satisfy the interchange rules for anyons:

$$
F\left(\ldots, \bar{\zeta}_{i}, \ldots\right)=P_{L}\left(\ldots, \bar{\zeta}_{i}, \ldots\right) \prod_{j<l}\left(\bar{\zeta}_{j}-\bar{\zeta}_{l}\right)^{\alpha}
$$

where $P_{L}$ is a symmetric polynomial of degree $L$ in the variables $\bar{\zeta}_{i}$. Note that for $\mathbf{k}=\mathbf{0}$ the problem has rotational symmetry about the $z$ axis, and the degree of the symmetric polynomial $L$ is related to the exciton angular momentum $\left[L_{z}=-L-N(N-1) \alpha / 2\right]$. We are now in a position to express the anyon exciton basis functions in the final form

$$
\begin{aligned}
\Psi\left(\mathbf{R}, \boldsymbol{\rho},\left\{\zeta_{i}\right\}\right)= & \exp \left\{i \mathbf{k} \cdot \mathbf{R}+i \hat{\mathbf{z}} \cdot[\mathbf{R} \times \boldsymbol{\rho}] / 2-(\boldsymbol{\rho}-\mathbf{d})^{2} / 4\right\} \\
& \times P_{L}\left(\ldots, \bar{\zeta}_{i}, \ldots\right) \\
& \times \prod_{j<l}\left(\bar{\zeta}_{j}-\bar{\zeta}_{l}\right)^{\alpha} \prod_{p} \exp \left\{-\left|\zeta_{p}\right|^{2} / 4 N\right\}
\end{aligned}
$$

\section{Symmetric polynomials}

We now consider the precise structure of the symmetric polynomials $P_{L}$ that appear in Eq. (26). To determine the symmetric basis polynomials of order $L$ we apply the fundamental theorem of symmetric polynomials, ${ }^{17}$ which states that any symmetric polynomial in $N$ variables, $P\left(x_{1}, \ldots, x_{N}\right)$, can be uniquely expressed in terms of the elementary symmetric polynomials 


$$
\begin{gathered}
\sigma_{1}=\sum_{i} x_{i}, \quad \sigma_{2}=\sum_{i<j} x_{i} x_{j}, \quad \sigma_{3}=\sum_{i<j<k} x_{i} x_{j} x_{k}, \\
\ldots, \quad \sigma_{N}=x_{1} x_{2} \cdots x_{N} .
\end{gathered}
$$

It is now apparent that all linearly independent symmetric basis polynomials of a particular degree may be enumerated by considering the possible products of the elementary symmetric polynomials (27), as the total degree $L$ is the sum of the degrees of the constituent polynomials. For example, the possible symmetric polynomials of degree four are constructed from $\sigma_{1}^{4}, \sigma_{1}^{2} \sigma_{2}, \sigma_{1} \sigma_{3}, \sigma_{2}^{2}$, and $\sigma_{4}$.

There are two further problems. The first is to determine the number of possible products of elementary symmetric polynomials for a total degree $L$. The second is to determine the structure of these products.

To calculate the number of linearly independent symmetric basis polynomials of degree $L$ which may be constructed from the elementary symmetric polynomials $\sigma_{1}, \ldots, \sigma_{N}$ we use a result from the theory of partitions. ${ }^{18}$

The number of ways of partitioning a number $L$ into parts of size $1,2, \ldots, P$ is given by the coefficient of $x^{L}$ in the expansion of

$$
\frac{1}{1-x} \frac{1}{1-x^{2}} \cdots \frac{1}{1-x^{P}}=\prod_{k=1}^{P} \frac{1}{1-x^{k}},
$$

where each term in the product is known as a generating function. The number of ways of partitioning increases rapidly with $L$ and does not follow any pattern. Furthermore, the different products of elementary symmetric polynomials for a particular value of $L$ must be determined by hand. An approximate formula for calculating the number of ways of partitioning a number does exist, the so-called HardyRamanujan formula, ${ }^{19}$ but it is not applicable to the present case as we have no polynomial of order one due to constraint (5). This constraint significantly reduces the number of possible symmetric polynomials by removing the first factor in the product (28). For example, for $N=3$ the number of polynomials of degree $L$ is equal to the integer part of $L / 6+1$ for even $L$ and the integer part of $(L-3) / 6+1$ for odd $L$, which corresponds to the result obtained in Ref. 2 .

\section{Discussion}

The key difference between the current general formulation and that outlined in Ref. 2 for the four-particle case $(N=3)$ is the replacement of anyon difference coordinates $\bar{z}_{j l}$ by the new coordinates $\bar{\zeta}_{i}$. The principal advantage of the difference coordinates was that they simplified the calculation of interanyon repulsion matrix elements, as well as the form of the statistical factor in the exciton wavefunction. However, they had the disadvantage that the classification of symmetric polynomials was more difficult, as it was necessary to introduce a Vandermonde determinant for odd- $L$ polynomials. For example, in the simplest case $L=3$, even though the number of anyon coordinates is the same in both formulations, we have $\sigma_{3}=\left(\bar{z}_{12}-\bar{z}_{23}\right)\left(\bar{z}_{23}-\bar{z}_{31}\right)\left(\bar{z}_{31}-\bar{z}_{12}\right)$ in terms of difference coordinates, whereas we have a simple product $\sigma_{3}=\bar{\zeta}_{1} \bar{\zeta}_{2} \bar{\zeta}_{3}$ in terms of the new coordinates. For a number of anyons greater than three this disadvantage is crucial, as the classification of polynomials in terms of $\bar{z}_{j l}$ becomes too cumbersome and the number of constraints on these coordinates is greater than one.

\section{SIX-PARTICLE ANYON EXCITON: BOSON APPROXIMATION}

\section{A. Formulation}

We introduce an anyon exciton consisting of a hole and five anyons with charge $-e / 5$. We also make a boson approximation so that the statistical factor $\alpha=0$. Our justification for this step is as follows. It was shown in Ref. 2 that for large values of $h$ (which is required for the AEM to be valid) the statistical factor $\alpha$ becomes unimportant, and the results for $\alpha=0$ were very similar to those for $\alpha= \pm 1 / 3$. We would therefore also expect this to be true for $\alpha= \pm 1 / N$, as this is even closer to zero. From now on we consider a boson approximation $(\alpha=0)$ for anyon statistics.

The explicit form of the exciton basis functions is

$$
\begin{aligned}
\Psi_{L, M, \mathbf{k}}\left(\mathbf{R}, \boldsymbol{\rho},\left\{\zeta_{i}\right\}\right)= & \exp \left\{i \mathbf{k} \cdot \mathbf{R}+i \hat{\mathbf{z}} \cdot[\mathbf{R} \times \boldsymbol{\rho}] / 2-(\boldsymbol{\rho}-\mathbf{d})^{2} / 4\right\} \\
& \times P_{L, M}\left(\bar{\zeta}_{1}, \ldots, \bar{\zeta}_{5}\right) \\
& \times \prod_{j=1}^{5} \exp \left\{-\left|\zeta_{j}\right|^{2} / 20\right\}
\end{aligned}
$$

where $M$ enumerates different linearly independent symmetric polynomials of degree $L$. To construct the symmetric polynomials $P_{L, M}$ we need to consider only the elementary symmetric polynomials $\sigma_{2}, \sigma_{3}, \sigma_{4}$, and $\sigma_{5}$. Note that $\sigma_{1}$ $=0$ because of constraint (5). From the above, we find that the number of possible ways of constructing a polynomial of degree $L$ is therefore the coefficient of $x^{L}$ in the expansion

$$
\frac{1}{1-x^{2}} \frac{1}{1-x^{3}} \frac{1}{1-x^{4}} \frac{1}{1-x^{5}}=\prod_{k=2}^{5} \frac{1}{1-x^{k}} .
$$

The possible ways of constructing the first twelve polynomials are shown explicitly in Table I. It can be seen that the number and complexity of the polynomials increases rapidly with the degree $L$.

It is evident from Eq. (29) that basis functions with different values of $\mathbf{k}$ are orthogonal. We now expand a general exciton wave function for given $\mathbf{k}$ in terms of the complete set of basis functions (29):

$$
\Phi=\sum_{i} \chi_{i} \Psi_{i}
$$

We shall show in Sec. III B that functions with different $L$ are orthogonal. However, basis functions with the same value of $L$ but different $M$ are not necessarily orthogonal, so their scalar products $\left\langle L, M \mid L, M^{\prime}\right\rangle$ will be nonzero. We therefore write the Schrödinger equation in matrix form as

$$
\hat{H} \boldsymbol{\chi}=\varepsilon \hat{B} \boldsymbol{\chi}
$$


TABLE I. Possible ways of constructing a symmetric polynomial $P_{L}$ from the elementary symmetric polynomials $\sigma_{2}, \sigma_{3}, \sigma_{4}$, and $\sigma_{5}$.

\begin{tabular}{ccc}
\hline \hline $\begin{array}{c}\text { Order } \\
L\end{array}$ & $\begin{array}{c}\text { No. of } \\
\text { polynomials }\end{array}$ & Structure \\
\hline 0 & 1 & 1 \\
1 & 0 & - \\
2 & 1 & $\sigma_{2}$ \\
3 & 1 & $\sigma_{3}^{2}$ \\
4 & 2 & $\sigma_{2}^{2}, \sigma_{4}$ \\
5 & 2 & $\sigma_{2} \sigma_{3}, \sigma_{5}$ \\
6 & 3 & $\sigma_{2}^{3}, \sigma_{2} \sigma_{4}, \sigma_{3}^{2}$ \\
7 & 3 & $\sigma_{2}^{2} \sigma_{3}, \sigma_{2} \sigma_{5}, \sigma_{3} \sigma_{4}$ \\
8 & 5 & $\sigma_{2}^{4}, \sigma_{2}^{2} \sigma_{4}, \sigma_{2} \sigma_{3}^{2}, \sigma_{3} \sigma_{5}, \sigma_{4}^{2}$ \\
9 & 5 & $\sigma_{2}^{3} \sigma_{3}, \sigma_{2}^{2} \sigma_{5}, \sigma_{2} \sigma_{3} \sigma_{4}, \sigma_{3}^{3}, \sigma_{4} \sigma_{5}$ \\
10 & 7 & $\sigma_{2}^{5}, \sigma_{2}^{3} \sigma_{4}, \sigma_{2}^{2} \sigma_{3}^{2}, \sigma_{2} \sigma_{3} \sigma_{5}, \sigma_{2} \sigma_{4}^{2}, \sigma_{3}^{2} \sigma_{4}, \sigma_{5}^{2}$ \\
11 & 7 & $\sigma_{2}^{4} \sigma_{3}, \sigma_{2}^{3} \sigma_{5}, \sigma_{2}^{2} \sigma_{3} \sigma_{4}, \sigma_{2} \sigma_{3}^{3}, \sigma_{2} \sigma_{4} \sigma_{5}$, \\
& & $\sigma_{3}^{2} \sigma_{5}, \sigma_{3} \sigma_{4}^{2}$ \\
12 & 10 & $\sigma_{2}^{6}, \sigma_{2}^{4} \sigma_{4}, \sigma_{2}^{3} \sigma_{3}^{2}, \sigma_{2}^{2} \sigma_{3} \sigma_{5}, \sigma_{2}^{2} \sigma_{4}^{2}, \sigma_{2} \sigma_{3}^{2} \sigma_{4}$, \\
& & $\sigma_{2} \sigma_{5}^{2}, \sigma_{3}^{4}, \sigma_{3} \sigma_{4} \sigma_{5}, \sigma_{4}^{3}$ \\
\hline
\end{tabular}

where $\hat{B}$ is the block-diagonal matrix of scalar products (the overlap matrix) and $\varepsilon$ is an energy eigenvalue. The size of each block in $\hat{B}$ depends on the number of different wave functions with given $L$. For example, in the case of a sixparticle exciton the block corresponding to $L=12$ will be of size $10 \times 10$ (see Table I). Both the overlap matrix $\hat{B}$ and the Hamiltonian matrix $\hat{H}$ are diagonal in $\mathbf{k}$. Note that for $\mathbf{k}$ $=\mathbf{0}$ the matrix $\hat{H}$ takes the same block-diagonal form as $\hat{B}$ (as will be shown in Secs. III C and III D), and as a result the problem becomes exactly soluble.

We shall now proceed to evaluate the matrix elements in Eq. (32). As $\hat{H}$ and $\hat{B}$ are diagonal in $\mathbf{k}$, in what follows we consider only the matrix elements diagonal in k. Since all terms in the polynomial $P_{L, M}$ are of the form of a product of the coordinates $\bar{\zeta}_{j}$, we use the following functions in monomials in the matrix element calculations:

$$
\begin{aligned}
\Psi_{\{n\}, \mathbf{k}}\left(\mathbf{R}, \boldsymbol{\rho},\left\{\zeta_{i}\right\}\right)= & C \exp \{i \mathbf{k} \cdot \mathbf{R}+i \hat{\mathbf{z}} \cdot[\mathbf{R} \times \boldsymbol{\rho}] / 2 \\
& \left.-(\boldsymbol{\rho}-\mathbf{d})^{2} / 4\right\} \bar{\zeta}_{1}^{n_{1}} \bar{\zeta}_{2}^{n_{2}} \bar{\zeta}_{3}^{n_{3}} \bar{\zeta}_{4}^{n_{4}} \bar{\zeta}_{5}^{n_{5}} \\
& \times \prod_{j=1}^{5} \exp \left\{-\left|\zeta_{j}\right|^{2} / 20\right\}
\end{aligned}
$$

where $\{n\}$ denotes the set of quantum numbers $n_{1}$ to $n_{5}$, and the constant $C$ will be defined in Sec. III B. The basis functions in terms of symmetric polynomials of degree $L$ can be obtained as a linear combination of the monomial functions (33), and $n_{1}+n_{2}+n_{3}+n_{4}+n_{5}=L$.

We also take into account constraint (5) via the following transformation:

$$
\begin{aligned}
\delta\left(\boldsymbol{\xi}_{1}\right. & \left.+\boldsymbol{\xi}_{2}+\boldsymbol{\xi}_{3}+\boldsymbol{\xi}_{4}+\boldsymbol{\xi}_{5}\right) \\
& =\int \frac{d \mathbf{f}}{(2 \pi)^{2}} \exp \left\{i \mathbf{f} \cdot\left(\boldsymbol{\xi}_{1}+\boldsymbol{\xi}_{2}+\boldsymbol{\xi}_{3}+\boldsymbol{\xi}_{4}+\boldsymbol{\xi}_{5}\right)\right\}
\end{aligned}
$$

\section{B. Overlap matrix elements}

To calculate the overlap matrix $\hat{B}$, let us consider the scalar product of two monomial functions:

$$
\begin{aligned}
\left\langle\{n\} \mid\left\{n^{\prime}\right\}\right\rangle= & \int d \mathbf{R} \int d \boldsymbol{\rho} \int \frac{d \mathbf{f}}{(2 \pi)^{2}} \\
& \times \int d \boldsymbol{\xi}_{1} d \boldsymbol{\xi}_{2} d \boldsymbol{\xi}_{3} d \boldsymbol{\xi}_{4} d \boldsymbol{\xi}_{5} \bar{\Psi}_{\{n\}, \mathbf{k}} \Psi_{\left\{n^{\prime}\right\}, \mathbf{k}} \\
& \times \exp \left\{i \mathbf{f} \cdot\left(\boldsymbol{\xi}_{1}+\boldsymbol{\xi}_{2}+\boldsymbol{\xi}_{3}+\boldsymbol{\xi}_{4}+\boldsymbol{\xi}_{5}\right)\right\} .
\end{aligned}
$$

The integration over $\mathbf{R}$ and $\boldsymbol{\rho}$ gives a factor of $2 \pi A$, and this leaves the following:

$$
\left\langle\{n\} \mid\left\{n^{\prime}\right\}\right\rangle=C^{2}(2 \pi A) \int \frac{d \mathbf{f}}{(2 \pi)^{2}} \prod_{j=1}^{5} M_{n_{j} n_{j}^{\prime}}(\mathbf{f}),
$$

where

$$
M_{m m^{\prime}}(\mathbf{f})=\int d \boldsymbol{\xi} \zeta^{m} \bar{\zeta}^{m^{\prime}} \exp \left(-\xi^{2} / 10+i \mathbf{f} \cdot \boldsymbol{\xi}\right) .
$$

If we now make the substitution $\zeta=\xi \exp \left(i \phi_{\xi}\right)$, and let $\phi$ be the angle between $\mathbf{f}$ and $\boldsymbol{\xi}$, i.e.,

$$
\phi=\phi_{\xi}-\phi_{\mathbf{f}}
$$

we obtain

$$
\begin{aligned}
M_{m m^{\prime}}(\mathbf{f})= & e^{i\left(m-m^{\prime}\right) \phi_{\mathbf{f}}} \int_{0}^{2 \pi} d \phi \int_{0}^{\infty} d \xi \xi^{1+m+m^{\prime}} \\
& \times \exp \left\{i\left(m-m^{\prime}\right) \phi+i f \xi \cos \phi-\xi^{2} / 10\right\} .
\end{aligned}
$$

We can now make use of Bessel's integral: ${ }^{20}$

$$
\int_{0}^{2 \pi} d \phi e^{ \pm i\left(m-m^{\prime}\right) \phi+i f \xi \cos \phi}=2 \pi i^{\left|m-m^{\prime}\right|} J_{\left|m-m^{\prime}\right|}(f \xi),
$$

to reduce Eq. (39) to the form

$$
M_{m m^{\prime}}(\mathbf{f})=2 \pi i^{\left|m-m^{\prime}\right|} e^{i\left(m-m^{\prime}\right) \phi_{\mathbf{f}}} \mathcal{M}_{m m^{\prime}}(f),
$$

where

$$
\mathcal{M}_{m m^{\prime}}(f)=\int_{0}^{\infty} d \xi \xi^{1+m+m^{\prime}} e^{-\xi^{2} / 10} J_{\left|m-m^{\prime}\right|}(f \xi) .
$$

This can also be expressed ${ }^{21}$ in terms of a confluent hypergeometric function $\Phi(\beta, \gamma ; z)$ as 


$$
\begin{aligned}
\mathcal{M}_{m m^{\prime}}(f)= & \frac{2^{\left(m+m^{\prime}\right) / 2} 5^{\left(m+m^{\prime}\right) / 2+1} \Gamma\left(\max \left\{m, m^{\prime}\right\}+1\right)}{\left|m-m^{\prime}\right| !} \\
& \times t^{\left|m-m^{\prime}\right| / 2} \Phi\left(\max \left\{m, m^{\prime}\right\}\right. \\
& \left.+1,\left|m-m^{\prime}\right|+1 ;-t\right),
\end{aligned}
$$

where $t=5 f^{2} / 2$ and $\max \left\{m, m^{\prime}\right\}$ is the largest of the integers $m$ and $m^{\prime}$. Equation (43) can be simplified further if we apply the Kummer transformation

$$
\Phi(\beta, \gamma ;-t)=e^{-t} \Phi(\gamma-\beta, \gamma ; t),
$$

and note that $(\gamma-\beta)$ is always a nonpositive integer:

$$
\gamma-\beta=\left|m-m^{\prime}\right|-\max \left\{m, m^{\prime}\right\} .
$$

This means that $\Phi(\gamma-\beta, \gamma ; t)$ reduces to a polynomial, and $\Phi(\beta, \gamma ;-t)$ is then just a polynomial in $t$ multiplied by $e^{-t}$.

If we now perform the integration over $\phi_{\mathrm{f}}$ we obtain the final form of the overlap matrix elements

$$
\left\langle\{n\} \mid\left\{n^{\prime}\right\}\right\rangle=C^{2} A(2 \pi)^{5} \delta_{L L^{\prime}} \int_{0}^{\infty} d f f \prod_{j=1}^{5} i^{\left|n_{j}-n_{j}^{\prime}\right|} \mathcal{M}_{n_{j} n_{j}^{\prime}}(f),
$$

where $L=n_{1}+n_{2}+n_{3}+n_{4}+n_{5}$. Note that nothing depends on $\mathbf{k}$ in this expression. It is convenient to define the constant $C$ so that for $L=L^{\prime}=0$ the matrix element $\left\langle\{n\} \mid\left\{n^{\prime}\right\}\right\rangle$ $=\langle 0 \mid 0\rangle=1$. This yields

$$
C^{2}=\frac{1}{125(2 \pi)^{5} A} \text {. }
$$

In the above formulation we have only considered matrix elements in terms of monomial functions. As mentioned earlier, matrix elements in terms of symmetric polynomials can be easily constructed as a linear combination of the monomial matrix elements (46).

\section{Anyon-anyon interaction}

The anyon-anyon interaction has the form

$$
\hat{V}_{a a}=\frac{1}{25} \sum_{j<l} \frac{1}{\left|\xi_{j}-\boldsymbol{\xi}_{l}\right|} .
$$

We shall only calculate the matrix elements for the first term $\hat{V}_{12}$ as the others follow by analogy. We begin by taking the Fourier transform of Eq. (48):

$$
\hat{V}_{12}\left(\boldsymbol{\xi}_{1}, \boldsymbol{\xi}_{2}\right)=\frac{1}{25} \int \frac{d \mathbf{q}}{2 \pi q} \exp \left\{i \mathbf{q} \cdot\left(\boldsymbol{\xi}_{1}-\boldsymbol{\xi}_{2}\right)\right\}
$$

This gives a matrix element in terms of monomials

$$
\begin{aligned}
\left\langle\{n\}\left|\hat{V}_{12}\right|\left\{n^{\prime}\right\}\right\rangle= & \frac{1}{25} \int d \mathbf{R} \int d \boldsymbol{\rho} \int \frac{d \mathbf{f}}{(2 \pi)^{2}} \int \frac{d \mathbf{q}}{2 \pi q} \\
& \times \int d \boldsymbol{\xi}_{1} d \boldsymbol{\xi}_{2} d \boldsymbol{\xi}_{3} d \boldsymbol{\xi}_{4} d \boldsymbol{\xi}_{5} \bar{\Psi}_{\{n\}, \mathbf{k}} \Psi_{\left\{n^{\prime}\right\}, \mathbf{k}} \\
& \times \exp \left\{i \mathbf{f} \cdot\left(\boldsymbol{\xi}_{1}+\boldsymbol{\xi}_{2}+\boldsymbol{\xi}_{3}+\boldsymbol{\xi}_{4}+\boldsymbol{\xi}_{5}\right)\right\} \\
& \times \exp \left\{i \mathbf{q} \cdot\left(\boldsymbol{\xi}_{1}-\boldsymbol{\xi}_{2}\right)\right\}
\end{aligned}
$$

Following the procedure in Sec. III B yields

$$
\begin{aligned}
\left\langle\{n\}\left|\hat{V}_{12}\right|\left\{n^{\prime}\right\}\right\rangle & \\
= & \frac{C^{2} A(2 \pi)^{3}}{25} \prod_{j=1}^{5} i^{\left|n_{j}-n_{j}^{\prime}\right|} \int d \mathbf{f} \int d \mathbf{q} \exp \left\{i \phi_{+}\left(n_{1}-n_{1}^{\prime}\right)\right. \\
& \left.+i \phi_{-}\left(n_{2}-n_{2}^{\prime}\right)\right\} \exp \left\{i \phi _ { \mathrm { f } } \left[\left(n_{3}-n_{3}^{\prime}\right)+\left(n_{4}-n_{4}^{\prime}\right)\right.\right. \\
& \left.\left.+\left(n_{5}-n_{5}^{\prime}\right)\right]\right\} \mathcal{M}_{n_{1} n_{1}^{\prime}}\left(f_{+}\right) \mathcal{M}_{n_{2} n_{2}^{\prime}}\left(f_{-}\right) \prod_{j=3}^{5} \mathcal{M}_{n_{j} n_{j}^{\prime}}(f),
\end{aligned}
$$

where $\mathcal{M}_{m m^{\prime}}$ are as defined in Eq. (42), $\mathbf{f}_{ \pm}=\mathbf{f} \pm \mathbf{q}$, and $\phi_{ \pm}$ are the phases of $\mathbf{f}_{ \pm}$.

We now seek to eliminate $\phi_{\mathrm{f}}$ by the change of variables $\phi=\phi_{\mathrm{f}}-\phi_{\mathbf{q}}$ and $\psi_{ \pm}=\phi_{ \pm}-\phi_{\mathbf{f}}$. After substitution and integration over $\phi_{\mathbf{q}}$ we obtain

$$
\begin{aligned}
\left\langle\{n\}\left|\hat{V}_{12}\right|\left\{n^{\prime}\right\}\right\rangle= & \frac{C^{2} A(2 \pi)^{4}}{25} \delta_{L L^{\prime}} \\
& \times \prod_{j=1}^{5} i^{\left|n_{j}-n_{j}^{\prime}\right|} \int_{0}^{\infty} d f f \int_{0}^{\infty} d q \int_{0}^{2 \pi} d \phi \\
& \times \exp \left\{i \phi\left(L-L^{\prime}\right)\right\} \exp \left\{i \psi_{+}\left(n_{1}-n_{1}^{\prime}\right)\right. \\
& \left.+i \psi_{-}\left(n_{2}-n_{2}^{\prime}\right)\right\} \mathcal{M}_{n_{1} n_{1}^{\prime}}\left(f_{+}\right) \\
& \times \mathcal{M}_{n_{2} n_{2}^{\prime}}\left(f_{-}\right) \prod_{j=3}^{5} \mathcal{M}_{n_{j} n_{j}^{\prime}}
\end{aligned}
$$

where $L=n_{1}+n_{2}+n_{3}+n_{4}+n_{5}$, and $\psi_{ \pm}$and $f_{ \pm}$can be expressed in terms of the variables of integration as

$$
e^{i \psi_{ \pm}}=\left(f \pm q e^{-i \phi}\right) / f_{ \pm}, \quad f_{ \pm}^{2}=f^{2}+q^{2} \pm 2 f q \cos \phi .
$$

It is evident from Eq. (52) that the anyon-anyon interaction matrix has the same block-diagonal structure as the overlap matrix. The integrand in Eq. (52) can be further reduced to a product of a polynomial in $q, f$, and $e^{ \pm i \phi}$, and an exponential factor $\exp \left(-25 f^{2} / 2-5 q^{2}\right)$. This integral can therefore be evaluated analytically for any $\{n\}$ and $\left\{n^{\prime}\right\}$. For the case of $L=L^{\prime}=0$, using Eq. (47) for $C^{2}$, the anyon-anyon interaction matrix element reduces to

$$
\left\langle 0\left|\hat{V}_{a a}\right| 0\right\rangle=\frac{1}{5} \sqrt{\frac{\pi}{5}} .
$$




\section{Anyon-hole interaction}

The anyon-hole interaction takes the form

$$
\hat{V}_{a h}=-\frac{1}{5} \sum_{j=1}^{5} \hat{V}_{j h}=-\frac{1}{5} \sum_{j=1}^{5} \frac{1}{\left|\mathbf{r}_{j h}\right|}
$$

where

$$
\mathbf{r}_{j h}=\boldsymbol{\xi}_{j}-\boldsymbol{\rho}+h \hat{\mathbf{z}}
$$

Considering only $\hat{V}_{1 h}$, we take the Fourier transform:

$$
\hat{V}_{1 h}\left(\boldsymbol{\rho}, \boldsymbol{\xi}_{1}\right)=-\frac{1}{5} \int \frac{d \mathbf{q}}{(2 \pi)^{2}} V_{a h}(q) \exp \left\{i \mathbf{q} \cdot\left(\boldsymbol{\xi}_{1}-\boldsymbol{\rho}\right)\right\}
$$

where

$$
V_{a h}(q)=\frac{2 \pi}{q} e^{-q h}
$$

The matrix elements are then

$$
\begin{aligned}
\left\langle\{n\}\left|\hat{V}_{1 h}\right|\left\{n^{\prime}\right\}\right\rangle= & -\frac{1}{5} \int d \mathbf{R} \int d \boldsymbol{\rho} \int \frac{d \mathbf{f}}{(2 \pi)^{2}} \\
& \times \int \frac{d \mathbf{q}}{(2 \pi)^{2}} V_{a h}(q) \\
& \times \int d \boldsymbol{\xi}_{1} d \boldsymbol{\xi}_{2} d \boldsymbol{\xi}_{3} d \boldsymbol{\xi}_{4} d \boldsymbol{\xi}_{5} \bar{\Psi}_{\{n\}, \mathbf{k}} \Psi_{\left\{n^{\prime}\right\}, \mathbf{k}} \\
& \times \exp \left\{i \mathbf{f} \cdot\left(\boldsymbol{\xi}_{1}+\boldsymbol{\xi}_{2}+\boldsymbol{\xi}_{3}+\boldsymbol{\xi}_{4}+\boldsymbol{\xi}_{5}\right)\right\} \\
& \times \exp \left\{i \mathbf{q} \cdot\left(\boldsymbol{\xi}_{1}-\boldsymbol{\rho}\right)\right\}
\end{aligned}
$$

Integration over $\mathbf{R}$ and $\boldsymbol{\rho}$ gives $2 \pi A \exp \left(-q^{2} / 2-i \mathbf{d} \cdot \mathbf{q}\right)$. Then, following the procedure in Section III $B$ once again, we obtain

$$
\begin{aligned}
\left\langle\{n\}\left|\hat{V}_{1 h}\right|\left\{n^{\prime}\right\}\right\rangle= & -\frac{C^{2} A(2 \pi)^{2}}{5} \prod_{j=1}^{5} i^{\left|n_{j}-n_{j}^{\prime}\right|} \int d \mathbf{f} \\
& \times \int d \mathbf{q} V_{a h}(q) e^{-q^{2} / 2-i \mathbf{d} \cdot \mathbf{q}} \\
& \times \exp \left\{i \phi_{+}\left(n_{1}-n_{1}^{\prime}\right)+i \phi_{\mathbf{f}}\left[\left(L-L^{\prime}\right)\right.\right. \\
& \left.\left.-\left(n_{1}-n_{1}^{\prime}\right)\right]\right\} \mathcal{M}_{n_{1} n_{1}^{\prime}}\left(f_{+}\right) \prod_{j=2}^{5} \mathcal{M}_{n_{j} n_{j}^{\prime}}(f),
\end{aligned}
$$

where $\mathcal{M}_{m m^{\prime}}$ are as defined in Eq. (42), $\mathbf{f}_{+}=\mathbf{f}+\mathbf{q}$, and $\phi_{+}$is the phase of $\mathbf{f}_{+}$. As before, $L=n_{1}+n_{2}+n_{3}+n_{4}+n_{5}$.

We now eliminate $\phi_{\mathbf{f}}$ by substituting $\phi=\phi_{\mathbf{f}}-\phi_{\mathbf{q}}$ and $\psi$ $=\phi_{+}-\phi_{\mathbf{f}}$. Choosing the $x$ axis along $\mathbf{d}$, the integration over $\phi_{\mathbf{q}}$ is then

$$
\begin{gathered}
\int_{0}^{2 \pi} d \phi_{\mathbf{q}} \exp \left\{i \phi_{\mathbf{q}}\left(L-L^{\prime}\right)-i d q \cos \phi_{\mathbf{q}}\right\} \\
=2 \pi(-i)^{\left|L-L^{\prime}\right|_{\left|L-L^{\prime}\right|}(d q),}
\end{gathered}
$$

which gives for the matrix elements

$$
\begin{aligned}
\left\langle\{n\}\left|\hat{V}_{1 h}\right|\left\{n^{\prime}\right\}\right\rangle= & -\frac{C^{2}(2 \pi)^{4} A}{5}(-i)^{\left|L-L^{\prime}\right|} \prod_{j=1}^{5} i^{\left|n_{j}-n_{j}^{\prime}\right|} \\
& \times \int_{0}^{\infty} d q e^{-q^{2} / 2-q h} J_{\left|L-L^{\prime}\right|}(d q) \\
& \times \int_{0}^{\infty} d f f \int_{0}^{2 \pi} d \phi \exp \left\{i \phi\left(L-L^{\prime}\right)\right. \\
& \left.+i \psi\left(n_{1}-n_{1}^{\prime}\right)\right\} \mathcal{M}_{n_{1} n_{1}^{\prime}}\left(f_{+}\right) \prod_{j=2}^{5} \mathcal{M}_{n_{j} n_{j}^{\prime}}(f),
\end{aligned}
$$

where $\psi$ and $f_{+}$can be expressed in terms of the variables of integration as

$$
e^{i \psi}=\left(f+q e^{-i \phi}\right) / f_{+}, \quad f_{+}^{2}=f^{2}+q^{2}+2 f q \cos \phi .
$$

For $d=0$, the Bessel function $J_{\left|L-L^{\prime}\right|}(d q)$ entering the integrand of Eq. (62) is nonzero only if $L=L^{\prime}$. This indicates that for the case of zero in-plane momentum $(k=d=0)$, the anyon-hole interaction matrix has the same block-diagonal structure as the anyon-anyon and overlap matrices. Therefore, states with different angular momentum $L_{z}$ decouple.

Equation (62) may be simplified further, and this yields the following expression for the anyon-hole interaction matrix elements for basis functions in terms of symmetric polynomials:

$$
\begin{aligned}
\left\langle L, M\left|\hat{V}_{a h}\right| L^{\prime}, M^{\prime}\right\rangle= & -\int_{0}^{\infty} d q \exp \left(-5 q^{2} / 2-q h\right) \\
& \times J_{\left|L-L^{\prime}\right|}(d q) Q_{L M, L^{\prime} M^{\prime}}(q),
\end{aligned}
$$

where $Q_{L M, L^{\prime} M^{\prime}}(q)$ is a polynomial in $q$ (the lowest order polynomial is $Q_{00,00}=1$ ). A similar expression to Eq. (64) is obtained for a four-particle exciton in Ref. 2. For $h=0$, Eq. (64) further reduces to an expression in terms of elementary functions. For the case of $k=d=0$ and $L=L^{\prime}=0$, the anyon-hole interaction matrix element is then

$$
\left\langle 0\left|\hat{V}_{a h}\right| 0\right\rangle=-\sqrt{\frac{\pi}{10}}
$$

\section{E. Exact results for $\mathrm{k}=0, L=0$}

All the above results are simplified significantly for the state with $\mathbf{k}=\mathbf{0}$ and $L=0$. For such a state, the $(N+1)$-particle wave function (26) with $\alpha=0$ reduces to the form 


$$
\begin{aligned}
\Psi\left(\mathbf{R}, \boldsymbol{\rho},\left\{\zeta_{i}\right\}\right)= & C_{N} \exp \left\{i \hat{\mathbf{z}} \cdot[\mathbf{R} \times \boldsymbol{\rho}] / 2-\rho^{2} / 4\right\} \\
& \times \exp \left\{-\sum_{p=1}^{N}\left|\zeta_{p}\right|^{2} / 4 N\right\},
\end{aligned}
$$

where the normalization constant $C_{N}$ can be determined from the integral

$$
\begin{aligned}
& C_{N}^{2} \int d \mathbf{R} \int \frac{d \mathbf{f}}{(2 \pi)^{2}} \int d \boldsymbol{\rho} e^{-\rho^{2} / 2} \int d \boldsymbol{\xi}_{1} \cdots d \boldsymbol{\xi}_{N} \\
& \quad \times \exp \left\{\sum_{p=1}^{N}\left(i \boldsymbol{\xi}_{p} \cdot \mathbf{f}-\xi_{p}^{2} / 2 N\right)\right\}=\frac{C_{N}^{2} A}{N^{2}}(2 \pi N)^{N}=1 .
\end{aligned}
$$

For the case of $\mathbf{k}=\mathbf{0}$ and $L=0$, the interaction matrix elements of a $(N+1)$-particle exciton are straightforward to evaluate. The anyon-anyon matrix element is

$$
\begin{aligned}
\left\langle 0\left|\hat{V}_{a a}\right| 0\right\rangle= & \frac{N(N-1)}{2 A(2 \pi N)^{N}} \int d \mathbf{R} \\
& \times \int \frac{d \mathbf{f}}{(2 \pi)^{2}} \int \frac{d \mathbf{q}}{2 \pi q} \int d \boldsymbol{\rho} e^{-\rho^{2} / 2} \int d \boldsymbol{\xi}_{1} \cdots d \boldsymbol{\xi}_{N} \\
& \times \exp \left\{\sum_{p=1}^{N}\left(i \boldsymbol{\xi}_{p} \cdot \mathbf{f}-\xi_{p}^{2} / 2 N\right)+i \mathbf{q} \cdot\left(\boldsymbol{\xi}_{1}-\boldsymbol{\xi}_{2}\right)\right\} \\
= & \frac{(N-1)}{4 N} \sqrt{\frac{\pi}{N}} .
\end{aligned}
$$

For nonzero interplane separation $h$ the anyon-hole matrix element can be reduced to

$$
\begin{aligned}
\left\langle 0\left|\hat{V}_{a h}\right| 0\right\rangle= & -\frac{N^{2}}{A(2 \pi N)^{N}} \int d \mathbf{R} \\
& \times \int \frac{d \mathbf{f}}{(2 \pi)^{2}} \int \frac{d \mathbf{q}}{2 \pi q} \int d \boldsymbol{\rho} e^{-\rho^{2} / 2} \int d \boldsymbol{\xi}_{1} \cdots d \boldsymbol{\xi}_{N} \\
& \times \exp \left\{\sum_{p=1}^{N}\left(i \boldsymbol{\xi}_{p} \cdot \mathbf{f}-\xi_{p}^{2} / 2 N\right)+i \mathbf{q} \cdot\left(\boldsymbol{\xi}_{1}-\boldsymbol{\rho}\right)-q h\right\} \\
= & -\int_{0}^{\infty} d q \exp \left(-N q^{2} / 2-q h\right) \\
= & -\sqrt{\frac{\pi}{2 N} e^{h^{2} / 2 N} \operatorname{erfc}(h / \sqrt{2 N}),}
\end{aligned}
$$

where $\operatorname{erfc}(x)$ is the complementary error function. Using the asymptotic expansion of $\operatorname{erfc}(x)$ it can be easily seen from Eq. (69) that $\left\langle 0\left|\hat{V}_{a h}\right| 0\right\rangle \rightarrow-1 / h$ as $h \rightarrow \infty$, as expected.

Equations (68) and (69) also allow us to calculate the critical interplane separation $h_{c}$ at which the $\mathbf{k}=\mathbf{0}, L=0$ state becomes unbound, i.e., when

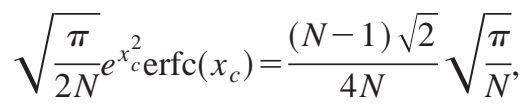

where $x_{c}=h_{c} / \sqrt{2 N}$. So, for $N=3$ the critical separation $h_{c}$ $\approx 5.39 l_{H}$, for $N=5$ we find that $h_{c} \approx 5.59 l_{H}$, and for $N \gg 1$ we have $h_{c} \approx 1.32 \sqrt{2 N} l_{H}$. Notably, these critical separations are well inside the region for which the AEM is applicable. It should be emphasized that the state with $L=0$ is not the ground state for the anyon exciton at large separation $h$. For example, for a four-particle exciton, ${ }^{2}$ the ground states for large separation satisfy a superselection rule $L=3 m$, where $m$ is an integer, and when $h \rightarrow \infty$ the ground state energy tends to its classical value, $\varepsilon=-(2 / 3)^{3 / 2} / h$.

Despite the nonapplicability of our model to a real physical situation at small layer separations $\left(h<l_{H}\right)$, the model remains soluble for all values of $h$, including $h=0$. Moreover, it has been shown in Ref. 2 that the ground state of the four-particle problem $(N=3)$ at $h=0$ is the state with $\mathbf{k}$ $=\mathbf{0}$ and $L=0$. We expect the same to be true for $N \geqslant 5$, since the anyon-hole attraction will always overcome the anyonanyon repulsion at small interparticle separations. It can be shown (in a similar way to that in Ref. 2) that the smallest average interparticle separation corresponds to the $L=0$ case. Therefore, the case of $\mathbf{k}=\mathbf{0}, L=0$ is of special interest since it predicts the anyon exciton binding energies in the limit $h \rightarrow 0$.

We are now in a position to write down a general expression for the binding energy of a $(N+1)$-particle exciton at zero 2DEG-hole separation $(h=0)$ :

$$
E_{b}=-\left(\left\langle 0\left|\hat{V}_{a h}\right| 0\right\rangle+\left\langle 0\left|\hat{V}_{a a}\right| 0\right\rangle\right)=\left[1-\frac{(N-1)}{2 \sqrt{2} N}\right] \sqrt{\frac{\pi}{2 N}}
$$

Equation (71) has been written in this particular form to emphasize the key result that for any value of $N$ there always exists at least one bound state of a neutral $(N+1)$-particle anyon exciton at $h=0$. For $N=1$, Eq. (71) yields the value $\sqrt{\pi / 2}$, which corresponds to that obtained in Ref. 16 for a standard diamagnetic exciton. For a four-particle exciton $(N=3), E_{b}=(1-\sqrt{2} / 6) \sqrt{\pi / 6}$, which agrees with the result of Ref. 2. Finally, for $N=5$ we have a binding energy of $(1-\sqrt{2} / 5) \sqrt{\pi / 10}$, which can also be obtained from Eqs. (54) and (65).

\section{CONCLUSIONS}

The anyon exciton model has been generalized to the case of an arbitrary number of anyons and several important mathematical results have been obtained. Starting from the Hamiltonian for a noninteracting system of $N$ anyons and a valence hole in a quantizing magnetic field, we have obtained a complete set of exciton basis functions. These functions have been fully classified using a result from the theory of partitions. We have derived expressions for the overlap and interaction matrix elements for a six-particle system, which describes an exciton against the background of an IQL 
with filling factor $\nu=2 / 5$. In the particular case of $\mathbf{k}=\mathbf{0}$ and $L=0$, we have found an expression for the binding energy of a $(N+1)$-particle exciton, which agrees with known results for a standard diamagnetic exciton and a four-particle anyon

*Also at A.F. Ioffe Physico-Technical Institute, St. Petersburg, Russia. Electronic address: m.e.portnoi@ex.ac.uk

${ }^{1}$ E.I. Rashba and M.E. Portnoi, Phys. Rev. Lett. 70, 3315 (1993).

${ }^{2}$ M.E. Portnoi and E.I. Rashba, Phys. Rev. B 54, 13791 (1996).

${ }^{3}$ B.B. Goldberg, D. Heiman, A. Pinczuk, C.W. Tu, A.C. Gossard, and J.H. English, Surf. Sci. 196, 209 (1988).

${ }^{4}$ D. Heiman, B.B. Goldberg, A. Pinczuk, C.W. Tu, A.C. Gossard, and J.H. English, Phys. Rev. Lett. 61, 605 (1988).

${ }^{5}$ A.J. Turberfield, S.R. Haynes, P.A. Wright, R.A. Ford, R.G. Clark, J.F. Ryan, J.J. Harris, and C.T. Foxon, Phys. Rev. Lett. 65, 637 (1990).

${ }^{6}$ V.M. Apalkov and E.I. Rashba, Solid State Commun. 95, 421 (1995).

${ }^{7}$ V.M. Apalkov, F.G. Pikus, and E.I. Rashba, Phys. Rev. B 52, 6111 (1995)

${ }^{8}$ F. Plentz, D. Heiman, A. Pinczuk, L.N. Pfeiffer, and K.W. West, Surf. Sci. 361/362, 30 (1996).

${ }^{9}$ F. Plentz, D. Heiman, A. Pinczuk, L.N. Pfeiffer, and K.W. West, Solid State Commun. 101, 103 (1997).

${ }^{10}$ G. Yusa, H. Shtrikman, and I. Bar-Joseph, Phys. Rev. Lett. 87, 216402 (2001). exciton. We have also shown that the $(N+1)$-particle exciton remains bound for 2DEG-hole separations exceeding several magnetic lengths, when the anyon exciton model becomes applicable to real physical systems.

${ }^{11}$ G. Yusa, H. Shtrikman, and I. Bar-Joseph, Physica E 12, 49 (2002).

${ }^{12}$ B.M. Ashkinadze, V. Voznyy, E. Cohen, A. Ron, and V. Umansky, Phys. Rev. B 65, 073311 (2002).

${ }^{13}$ M.K. Ellis, M. Hayne, A. Usher, A.S. Plaut, and K. Ploog, Phys. Rev. B 45, 13765 (1992).

${ }^{14}$ F. Bolton, Phys. Rev. Lett. 73, 158 (1994).

${ }^{15}$ L.P. Gor'kov and I.E. Dzyaloshinskii, Zh. Éksp. Teor. Fiz. 53, 717 (1967) [Sov. Phys. JETP 26, 449 (1968)].

${ }^{16}$ I.V. Lerner and Y.E. Lozovik, Zh. Éksp. Teor. Fiz. 78, 1167 (1978) [Sov. Phys. JETP 51, 588 (1980)].

${ }^{17}$ G. Birkhoff and S. MacLane, A Survey of Modern Algebra, 4th ed. (Macmillan, New York, 1977).

${ }^{18}$ A. Slomson, An Introduction to Combinatorics (Chapman and Hall, London, 1991).

${ }^{19}$ G.H. Hardy and S. Ramanujan, Proc. London Math. Soc. 2, 75 (1918).

${ }^{20}$ G. Arfken, Mathematical Methods for Physicists, 3rd ed. (Academic, London, 1985).

${ }^{21}$ I. Gradshteyn and I. Ryzhik, Table of Integrals, Series, and Products, 6th ed. (Academic, San Diego, 2000). 